\title{
Ação do gel Anacardium Occidentale L. associado ao ultrassom terapêutico no processo de cicatrização em camundongos
}

\section{Action of the gel Anacardium Occidentale L. associated with therapeutic ultrasound in the healing process in mice}

Renata Andressa Alves Furtado, Manoela Laís Pereira Noleto, Diego Rodrigues Pessoa, Vaniele da Silva Almeida, Antônio Luiz Martins Maia Filho, Valdiléia Teixeira Uchôa, Wellington dos Santos Alves

Como citar este artigo: FURTADO, R. A. A.; NOLÊTO, M. L. P.; PESSOA, D. R.; ALMEIDA, V. S.; MAIA FILHO, A. L. M.; UCHOA, V. T.; ALVES, W. S. Ação do gel Anacardium Occidentale L. associado ao ultrassom terapêutico no processo de cicatrização em camundongos. Revista Saúde (Sta. Maria). 2019; 45 (2).

\section{Autor correspondente:}

Nome: Renata Andressa Alves Furtado

E-mail: renatha.92@hotmail.com Telefone: (86) 98828-9998

Formação Profissional: Formada em Fisioterapia pela Universidade Estadual do Piauí (UESPI). Especialista em Fisioterapia dermatofuncional pela faculdade Inspirar, Teresina, Piauí, Brasil.

Filiação Institucional: Universidade Estadual do Piauí (UESPI) Endereco para correspondência: Rua: Olavo Bilac n: 2335 Bairro: Centro (Sul)

Cidade: Teresina Estado: Piauí CEP: 64001-280

Data de Submissão:

$05 / 11 / 2018$

Data de aceite:

04/08/2019

Conflito de Interesse: Não há conflito de interesse

\section{(cc) $\mathrm{BY}-\mathrm{NC}-\mathrm{ND}$}

\section{RESUMO}

INTRODUÇÃO: Os recursos fisioterapêuticos influenciam positivamente no processo de cicatrização, acelerando as diferentes fases e reduzindo o tempo de lesão através da aplicação de recursos eletrofísicos, tais como ultrassom terapêutico (UST). A interação entre o UST e os tecidos biológicos, é capaz de gerar alterações celulares, por efeitos mecânicos, garantindo resultados satisfatórios. Estudos à base dos extratos vegetais vêm sendo realizados visando comprovar terapias alternativas efetivas capazes de induzir a cicatrização de feridas, uma vez que as plantas medicinais quando comparadas com as modernas terapias medicamentosa, apresentam menores efeitos adversos e baixo custo, quando comparado aos fármacos sintéticos, sendo essenciais para aquisição para a população de baixa renda. OBJETIVO: Avaliar o efeito associado entre 0 gel à base da casca do cajueiro (Anacardium occidentale L) e o ultrassom terapêutico sobre o processo de cicatrização de feridas cutâneas em camundongos. METODOLOGIA: 24 camundongos (Mus musculus) machos, pesando entre 40 a 50 gramas $(\mathrm{g})$, provenientes do biotério da Faculdade de Ciências Médicas (FACIME), foram utilizados neste estudo. A pesquisa foi composta por 4 grupos (controle, ultrassom pulsado + fonorose, gel à base de cascas da Anacardium occidentale $L$ e ultrassom pulsado), os quais foram tratados em diferentes tempos experimentais ( 8 e 15 dias). Após a eutanásia dos camundongos, realizou-se as análises macroscópica e microscópica, enquanto 0 extrato vegetal à base do caule do cajueiro (Anacardium occidentale L) foi avaliado através da prospecção fitoquímica. RESULTADOS: Observa-se que tanto na análise histológica quanto macroscópica, houve melhora do processo inflamatório inicial e aceleração do reparo tecidual nos grupos tratados com o UST, extrato, assim como, através da sua interação. CONCLUSÃO: Conclui-se que as terapias utilizadas no presente estudo, apresentaram resultados satisfatórios sobre o processo de reparação tecidual, os quais foram comprovados pela análise macroscópica e histológica.

PALAVRAS-CHAVE: Terapia por Ultrassom; Medicamentos Fitoterápicos; Cicatrização de Feridas.

\section{ABSTRACT}

INTRODUCTION: Physical therapy resources positively influence the healing process, accelerating the different phases and reducing the time of injury through the application of electrophysical resources, such as therapeutic ultrasound (TUS). The interaction between TUS and biological tissues is capable of generating cellular changes by mechanical effects, ensuring satisfactory results. Studies based on plant extracts have been carried out to prove effective alternative therapies capable of inducing wound healing, since medicinal plants, when compared to modern drug therapies, have lower adverse effects and lower cost when compared to synthetic drugs, essential for acquisition for the low-income population. OBJECTIVE: To evaluate the associated effect between cashew bark gel (Anacardium occidentale L) and therapeutic ultrasound on the healing process of skin wounds in mice. METHODOLOGY: 24 male (Mus musculus) mice weighing 40 to 50 grams (g) from the Faculty of Medical Sciences (FACIME) 
were used in this study. The research consisted of 4 groups (control, pulsed ultrasound + phonorosis, Anacardium occidentale $L$ bark gel and pulsed ultrasound), which were treated at different experimental times (8 and 15 days). RESULTS: It was observed that both histological and macroscopic analysis showed improvement of the initial inflammatory process and acceleration of tissue repair in the groups treated with UST, extract, as well as through their interaction. CONCLUSION: It is concluded that the therapies used in the present study presented satisfactory results on the tissue repair process, which were confirmed by macroscopic and histological analysis.

\section{INTRODUÇÃO}

A pele, é considerada o maior órgão do corpo, composta pela epiderme, derme subjacente e seus anexos, responsável por funções importantes, tais como: a proteção, recepção de sensações contínuas do ambiente por meio dos receptores específicos da regulação da temperatura, absorção da radiação ultravioleta e a excreção através das glândulas sudoríparas ${ }^{1}$. O processo de cicatrização de feridas consiste no restabelecimento da continuidade tecidual, de um tecido cutaneomucoso agredido, através de eventos celulares, fisiológicos, moleculares e bioquímicos que interagem para que ocorra a restauração tissular. O processo de reparação tecidual é constituído por três fases distintas: inflamatória, proliferativa (granulação) e remodeladora (maturação) $)^{2,3}$.

A fase inflamatória é iniciada após injúria tecidual, mediante a liberação de substâncias vasoconstritoras (tromboxana A2 e prostaglandinas), no intuito de formar a cascata de granulação, cujo qual é responsável por estimular a liberação de plaquetas, as quais contêm fator de crescimento de transformação beta (TGF- $\beta$ ), fator de crescimento derivado das plaquetas (PDGF), fator de crescimento derivado dos fibroblastos (FGF), fator de crescimento epidérmico (EGF), que atraem neutrófilos à ferida, promovendo a quimiotaxia (migração de neutrófilos para a ferida) ${ }^{4,5}$. A fase proliferativa inicia-se ao fim do $4^{\circ}$ dia e se estende aproximadamente até o término da segunda semana ${ }^{4,5}$. É marcada pela proliferação intensa de fibroblastos, síntese de colágeno (tipo III) e matriz extracelular ${ }^{4,5}$. A fase remodeladora é caracterizada pela reabsorção de um colágeno inicial (tipo III) e, substituído, por um tipo de colágeno mais espesso (tipo I), cujo qual é mais organizado ao longo das linhas de tensão, favorecendo aumento da força tênsil da ferida ${ }^{4,5}$.

Diversos recursos terapêuticos têm sido estudados no intuito de reduzir o tempo de cicatrização, tais como: a fotobiomodulação (FBM) por laser de baixa intensidade (LBI) ${ }^{2}$ elou luz diodo emissor (LED) ${ }^{3}$, ondas curtas (modo pulsado) ${ }^{6}$, alta frequência ${ }^{7}$, microcorrentes ${ }^{8}$, atualmente, o ultrassom terapêutico (UST) vêm sendo estudo para promover a melhora do reparo tecidual ${ }^{9,15}$.

O UST é um recurso amplamente utilizado para acelerar o processo de reparo tecidual de feridas cutâneas, em que as ondas sonoras produzem vibrações mecânicas nas células do corpo, possibilitando produção de calor no tecido, fato que estará associado às respostas terapêuticas essenciais junto às células ${ }^{9}$. Entre os eventos fisiológicos relacionados à resposta inflamatória através do emprego da UST, destaca-se: a degranulação dos mastócitos, histamina 
e leucócitos, redução do edema, aumento do metabolismo celular e síntese de colágeno tipo I e III; melhor alinhamento e disposição das fibras de colágeno, que estão associados com a melhora efetiva do processo de reparo ${ }^{\text {. }}$

O UST permite a interação entre as ondas ultrassónicas com meios farmacológicos (fonoforese), como os fitoterápicos, através da produção do gel ou pelo uso de medicamento tópico, favorecendo os efeitos terapêuticos. No entanto, para a terapia apresentar resultados benéficos, é necessário adequar corretamente os parâmetros dosimétricos, como: o modo de acoplamento ${ }^{10}$, intensidade ${ }^{11}$, emissão ${ }^{12}$, tempo de aplicação $0^{13}$ e frequência ${ }^{14,15}$, adaptadas de acordo com o estágio da lesão $0^{2,3}$.

Além dos recursos eletrotermofototerapêuticos, vários estudos à base dos extratos vegetais vêm sendo realizados no intuito de comprovar terapias alternativas capazes de induzir a cicatrização de feridas ${ }^{16}$. É de suma importância salientar que as plantas medicinais quando comparadas com as modernas terapias medicamentosa, como antibióticos e corticoides, apresentam menores efeitos adversos e interações medicamentosas, assim como, baixo custo quando comparado aos fármacos sintéticos, sendo essenciais para aquisição para a população de baixa renda e, esses quando empregados de maneira efetiva, tem como objetivo promover a melhora da saúde e o bem-estar dos indivíduos ${ }^{16}$.

Várias plantas medicinais apresentam papel cicatrizante, uma vez que apresentam capacidade de atividade fibrinolítica, acelerando a formação do tecido de granulação, biossíntese de colágeno e elastina, entre as quais podem destacar as seguintes: mamona (Ricinus communis L. $)^{17}$, arnica (Arnica montana L.) $)^{18}$, babosa (Aloe vera L. $)^{19}$, folha santa (Bryophyllum calycinum Salisb. ${ }^{20}$, Capuchinha (Tropaeolum majus) ${ }^{21}$, Copaíba (Copaífera multijuga) $)^{22}$ e, recentemente, o gel a base do caule do cajueiro (Anacardium occidentale $L$ ) tem sido alvo de pesquisadores no intuito de comprovar a sua eficácia acerca do processo de reparo tecidual23,24.

A utilização de plantas medicinais (fitoterápicos) tem sido amplamente estudada nas áreas de atuação biomédicas, pois se trata de um método inovador e sofisticado para o tratamento de afeç̧ões patológicas, sendo que sua administração permite a promoção, preservação, manutenção e recuperação da saúde funcional dos indivíduos ${ }^{23,24}$. A. occidentale $L$ pertence à família Anacardiaceae muito abundante na região do Nordeste brasileiro ${ }^{23,24}$. É uma planta nativa do Brasil, que é composta pela castanha de caju (verdadeiro fruto) e o caju (pseudofruto), que apresenta excelentes propriedades nutricionais e sensoriais ${ }^{23,24}$.

Os extratos do caule e cascas da $A$. occidentale $L$, são amplamente utilizados para o tratamento de doenças respiratórias (bronquite e tosse), disfunções gastrointestinais (diarreia, disenteria, cólica intestinal e dor no cólon), assim como, evidencias apontam que o gel a base do extrato do caule tem apresentado propriedades antidiabéticas, antibacterianas, anti-inflamatórias e antiulcerogênicas ${ }^{23,24}$. Assim, este trabalho teve como objetivo avaliar o efeito associado entre o gel à base da casca do cajueiro (Anacardium occidentale $L$ ) e o ultrassom terapêutico sobre o processo de cicatrização de feridas cutâneas em camundongos. 


\section{MÉTODOS}

Aspectos éticos e legais

O projeto de pesquisa foi submetido à apreciação do Comitê de Ética em Pesquisa de Animais (CEUA) da Universidade Estadual do Piauí (UESPI) e obteve aprovação, sob protocolo nº 05711/15, seguindo os princípios éticos da experimentação animal, de acordo com as Normas Internacionais de Proteção aos Animais e do Colégio Brasileiro de Experimentação Animal (COBEA).

\section{Delineamento do estudo}

Foram estudados 24 camundongos (Mus musculus) machos, pesando entre 40 a $50 \mathrm{~g}$, provenientes do biotério da Faculdade de Ciências Médicas (FACIME) da UESPI. Todos os animais receberam, por via subcutânea, um prétratamento com xilazina (relaxante muscular), na dose de $1,1 \mathrm{ml} / \mathrm{kg}$, aguardando-se 15 minutos para o procedimento anestésico $^{26,27}$. Subsequentemente foram submetidos à anestesia dissociativa com ketamina $(1,0 \mathrm{ml} / \mathrm{kg})$, em seguida procedeu-se com a tricotomia do local do procedimento cirúrgico, realização da assepsia da região com iodo polvidine (PVPI), 24 horas antes do procedimento cirúrgico $26,27$.

Os animais foram distribuídos e acondicionados aleatoriamente em caixas específicas para camundongos, mantidos desde o nascimento em um ciclo claro/escuro de 12/12 horas, com alimentação e água ad libitum (Tabela $1)^{26,27}$. Antes da incisão, as bordas da ferida foram demarcadas utilizando-se caneta hidrográfica e um círculo universal feito de papel resistente confeccionado especialmente para a limitação da incisão com diâmetro de $3,0 \mathrm{~cm}^{26}$. As feridas foram produzidas com bisturi e tesoura íris, retirando-se pele, tecido subcutâneo e gordura, preservando a integridade do tecido muscular ${ }^{26}$. Posterior à recuperação anestésica, os camundongos foram transferidos para gaiolas, observados e tratados diariamente até o termino do $8^{\circ}$ dia (eutanásia) e $15^{\circ}$ dia (eutanásia) 26,27 .

\section{Grupos experimentais}

O grupo 1 (controle) foi apenas lesionada, em que os camundongos ficaram apenas em observação ao longo do experimento nos determinados tempos de eutanásia ( $8^{\circ}$ e $15^{\circ}$ dias). 0 grupo 2 recebeu o tratamento associado entre o UST pulsado (fonoforese) + gel das cascas do cajueiro (A. occidentale). Foi utilizado o aparelho de US (Ibramed, Sonopulse), ajustado com os seguintes parâmetros: modo pulsado $10 \%$, frequência de $1 \mathrm{MHz}$, intensidade de $0,5 \mathrm{~W} /$ 
$\mathrm{cm}^{2}$, método de acoplamento direto com movimentos oscilatórios, ERA de $1 \mathrm{~cm}^{2}$, durante 2 minutos, tratados conforme o protocolo experimental ( $8^{\circ}$ e $15^{\circ}$ dias). 0 grupo 3 recebeu o tratamento baseado no uso do gel tópico das cascas do cajueiro (A. occidentale), aplicado diariamente durante os $8^{\circ}$ e $15^{\circ}$ dias de tratamento. 0 grupo 4 foi tratado apenas com o UST, de acordo com a seguinte dosimetria: modo pulsado 10\%, frequência de $1 \mathrm{MHz}$, com intensidade de $0,5 \mathrm{~W} / \mathrm{cm}^{2}$, método de acoplamento direto com movimentos oscilatórios, ERA de $1 \mathrm{~cm}^{2}$, durante 2 minutos, administrados durante 8 e 15 dias.

Posteriormente os experimentos (8 e 15 dias), procedeu-se ao sacrifício dos animais com aplicação de uma dose excessiva de anestesia (anestésico tiopental) $)^{26,27}$.

Tabela 1. Divisão dos grupos experimentais

\begin{tabular}{llll} 
Grupo & Subgrupo & Eutanásia & Tratamento \\
\hline G1 & $1 a(n=3)$ & 8 dias & Controle. \\
& $1 b(n=3)$ & 15 dias & \\
\hline G2 & $2 a(n=3)$ & 8 dias & Ultrassom pulsado (fonoforese) + gel \\
& $2 b(n=3)$ & 15 dias & das cascas do cajueiro (A. occidentale). \\
\hline G3 & $3 a(n=3)$ & 8 dias & Gel das cascas do cajueiro (A. occidentale), \\
& $3 b(n=3)$ & 15 dias & uso tópico. \\
\hline G4 & $4 a(n=3)$ & 8 dias & Ultrassom pulsado. \\
& $4 b(n=3)$ & 15 dias & \\
\hline
\end{tabular}

Fonte: Autores, 2018.

Produção do extrato à base da casca do cajueiro

Para a produção do extrato à base das cascas do cajueiro, foi realizada a coleta na zona rural da cidade de Teresina - PI, seguindo as coordenadas ( $5^{\circ} 02^{\prime} 42.3$ "S"; 42 $44^{\circ} 28.8$ "W"), visando evitar agentes poluentes nas amostras coletadas, em que foi estabelecido horário fixo para a realização das mesmas, em seguida as amostras foram expostas a secagem ao sol em temperatura ambiente de $36^{\circ} \mathrm{C}$, durante duas semanas, sendo que após a retirada de toda umidade elas passarão por um processo de trituração, através de um triturador industrial (JBM 30) com a capacidade de armazenamento de 2 litros (L), o qual resultou em um componente à base de pó ${ }^{29}$.

Posteriormente a etapa inicial, foi utilizado $500 \mathrm{~g}$ do pó das cascas do cajueiro que foi introduzido em um extrator de vidro com capacidade de $500 \mathrm{ml}$, em seguida, foi administrado $400 \mathrm{ml}$ de álcool etílico à $99.5 \%$, o qual foi agitado por cerca de 5 minutos, em que se obteve a mistura entre o soluto e solvente ${ }^{29}$. A mistura permaneceu em maceração por 10 dias, transcorrido o tempo de maceração, a solução foi retirada do recipiente e filtrada com auxílio de papel 
filme (qualitativo) e depositado em um Erlenmeyer com capacidade de $500 \mathrm{~mL}$, posteriormente a mesma solução foi armazenada em um balão volumétrico, até ser conduzida ao rotaevaporador, com temperatura $\left(50-60^{\circ} \mathrm{C}\right)$ e rotação (120-140 RPM), retirando-se todo o solvente, resultando em um soluto concentrado em forma de pasta, que foi sujeita a remoção da umidade remanescente através da capela de exaustão de gases durante 48 horas, finalizando o processo com um composto concentrado das cascas de cajueiro ${ }^{29}$.

Produção do gel à base das cascas do cajueiro

Para fabricação do gel, foi utilizada uma concentração de $5 \%$ do extrato alcoólico das cascas do cajueiro, foram utilizadas $10 \mathrm{~g}$ de extrato para $200 \mathrm{ml}$ de gel de carbopol, sendo que para solubilizar o extrato, foram utilizadas $5 \mathrm{ml}$ de etanol e por fim, a mistura em um recipiente, o qual obteve um $\mathrm{pH}=5^{30}$.

\section{Avaliação fitoquímica}

Para identificar as principais classes de compostos orgânicos presentes, relacionados ao poder cicatrizante da A. occidentale $L^{29}$. Foi realizada uma prospecção fitoquímica do extrato etanólico das cascas do caule do cajueiro. Foram feitos testes qualitativos para identificação das principais classes dos princípios ativos presentes ${ }^{29}$. Utilizando assim 9 tubos de ensaio, contendo $2 \mathrm{~mL}$ de extrato bruto dissolvido em álcool etílico. Foram realizados testes para taninos, flavonoides, esteróides/triterpenóides, carboidratos, alcaloides, alguns flavonóis (antocianinas, antocianidinas, flavonas, flavonóis, xantonas, chalconas e auronas e saponinas) $)^{29}$.

\section{Avaliação Macroscópica}

Após a eutanásia ( $8^{\circ}$ e $\left.15^{\circ} \mathrm{dia}\right)$, foi realizada a análise macroscópica das lesões. Os animais foram fixados em uma prancha cirúrgica e, examinou-se com auxílio de lupa, a presença crostas, secreções e cicatrizes hipertróficas. Já o tamanho da ferida residual no maior e menor eixo, foi mensurado com um paquímetro digital. Os resultados foram anotados e, em seguida, realizou-se o registro fotográfico, com câmera digital com zoom de 1,3 de aproximação fixada em tripé. A partir do registro fotográfico, calculou-se a área da ferida, utilizando-se a planigrafia digital, transferindo a imagem para o software (Auto Cad), após delimitar a periferia da lesão, demarcados os pontos pelo método poliline e realizado o cálculo da área ${ }^{31}$. A medida do maior diâmetro foi determinada usando o paquímetro digital transformando-a em medida digital ${ }^{31}$. 


\section{Avaliação histológica}

Os cortes histológicos foram corados com hematoxilina eosina (H.E.). Vinte e quatro lâminas, sendo três de cada animal alocados nos grupos estudados, foram avaliadas quanto a presença de fibroblastos, leucócitos e células mono e polimorfonucleares. A análise microscópica foi realizada anotando-se os achados de modo qualitativo e quantitativo. A avaliação quantitativa foi realizada com base em análise comparativa entre os dois períodos de observação (8 e 15 dias), através do software Image J. ${ }^{26,27}$.

\section{Análise estatística}

Todas as variáveis obtidas no estudo foram analisadas com o auxílio do programa GraphPad Prism®, versão 5.0. A normalidade dos dados foi verificada através do teste de Shapiro-Wilk, em seguida, realizou-se à análise de variância (ANOVA) para múltiplas comparações (intragrupos e intergrupos), com pós-teste de Tukey, considerando um nível de significância de $5 \%(p<0,05)$.

\section{RESULTADOS}

O estudo da triagem fitoquímica (Tabela 2) do extrato bruto etanólico da casca do cajueiro (A. occidentale, $L$ ) revelou resultados positivos para a taninos, flavanóides, triterpenóides, carboidratos, alcalóides, antocianidinas, chalconas e auronas ( $\mathrm{pH}=3)$, anitocianinas e antocianidinas $(\mathrm{pH}=8.5)$ e chalconas, auronas e flavonóis $(\mathrm{pH}=11)$. Foram observados testes negativos para esteroides, saponinas, flavononas e flavonóis, xantonas. No entanto, os resultados negativos em relação a esses compostos, não implicam necessariamente na sua ausência, sendo provável que a quantidade dos mesmos esteja pequena para ser detectada.

Tabela 1 - Dados da análise fitoquímica dos metabólitos secundários.

\begin{tabular}{cc}
\hline CLASSES DE COMPOSTOS ORGÂNICOS & EXTRATO DA CASCA DO CAULE DO CAJUEIRO \\
\hline Taninos & Positivo $(+)$ \\
\hline Flavonóides & Positivo $(+)$ \\
\hline Esteróides & Negativo $(-)$ \\
\hline Triterpenóides & Positivo $(+)$ \\
\hline Carboidratos & Positivo $(+)$ \\
\hline Alcalóides & Positivo $(+)$ \\
\hline Saponinas & Negativo $(-)$ \\
\hline
\end{tabular}




\begin{tabular}{|c|c|}
\hline \multirow{3}{*}{ Antocianinas e Antocianidinas } & pH 3: Positivo (+) \\
\hline & pH 8,5: Positivo (+) \\
\hline & pH 11: Negativo (-) \\
\hline Flavononas e Flavonóis & Negativo (-) \\
\hline Xantonas & Negativo (-) \\
\hline \multirow{3}{*}{ Chalconas e Auronas } & pH 3: Positivo (+) \\
\hline & pH 8,5: Negativo (-) \\
\hline & pH 11: Positivo (+) \\
\hline \multirow{3}{*}{ Flavonóis } & pH 3: Negativo (-) \\
\hline & pH 8,5: Negativo (-) \\
\hline & pH 11: Positivo (+) \\
\hline
\end{tabular}

Fonte: Autores, 2019.

A figura $A$, demonstra os valores obtidos acerca do diâmetro das feridas (pós-cirúrgico), em que foi possível verificar nenhuma diferença estatística entre diâmetro do corte nos grupos estudados. A figura B (8 dias) é observada diferenças estatísticas significativas (intergrupos) entre G1 e G3 ( $p<0.001$ ), G1 e G4 ( $p<0.001), G 2$ e G3 ( $p<0.001$ ) e G2 e G4 ( $p<0.001)$. A figura C (15 dias) demonstrou valores significativos entre as análises dos grupos $\mathrm{G} 1$ e $\mathrm{G} 2(\mathrm{p}<0.0001)$, G1 e $G 3(p<0.0001)$ e $G 1$ e $G 4(p<0.0001)$.
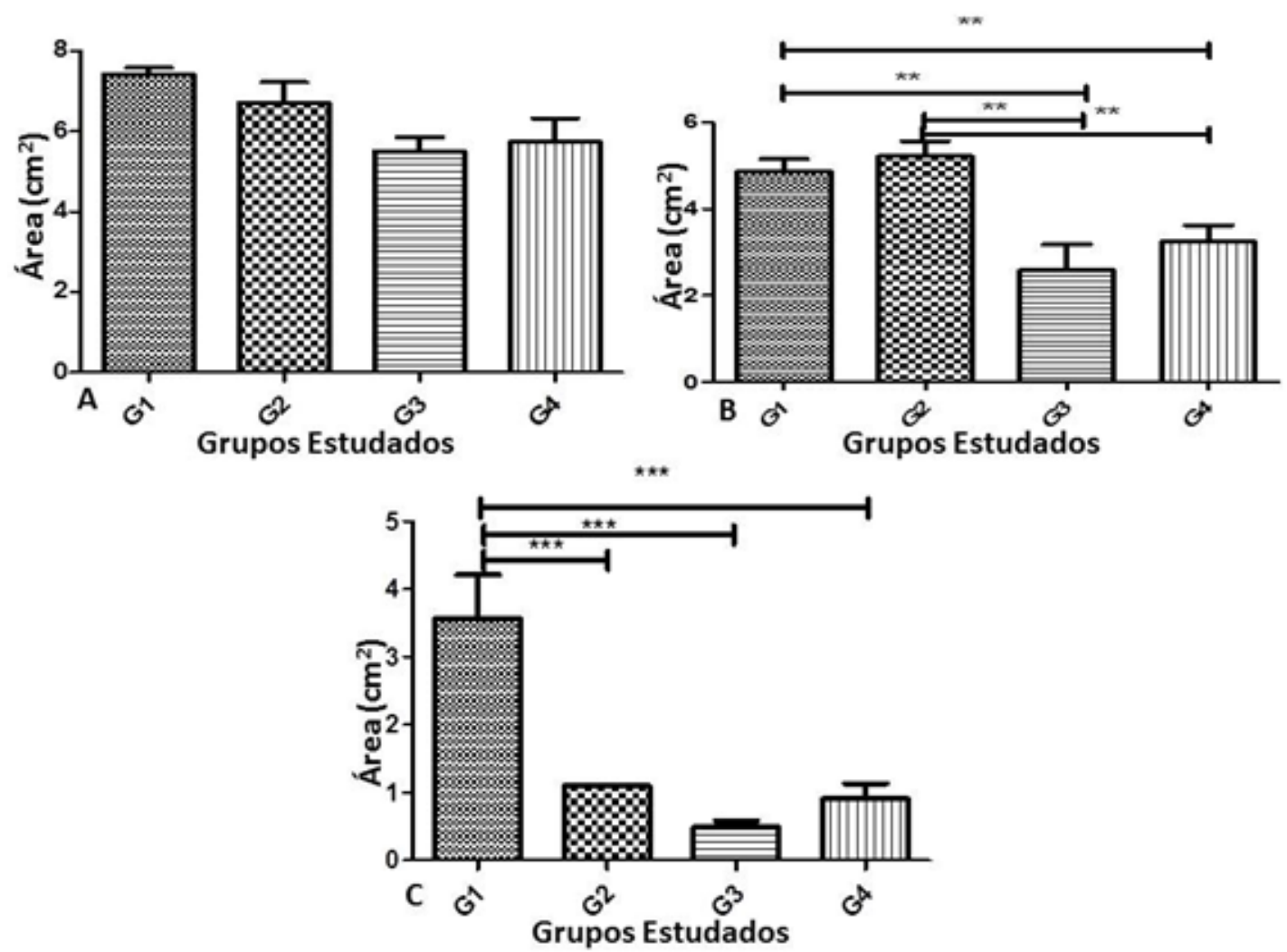

Figura 1. Média dos valores correspondendo às áreas das feridas em diferentes tempos experimentais: $A(0$ dia), B (7 dias) e C (15 dias). Legenda. G1 (Controle). G2 (Ultrassom pulsado (fonoforese) + gel das cascas do cajueiro (A. occidentale). G3 (Gel das cascas do cajueiro (A. occidentale), uso tópico). G4 (Ultrassom pulsado). Fonte: Autores, 2019. 
$\mathrm{Na}$ análise dos cortes histológicos (Figura 2), verificou-se, que na comparação entre 8 e 15 dias (análise intragrupos) G2 (p<0.05), G3 (p<0.001) e G4 (p<0.001) demonstraram redução expressiva no número de células inflamatórias. Já análise intergrupos (8 dias) evidenciou que não houve diferença significativa entre os tratamentos aplicados no tempo experimental proposto, enquanto que a avaliação intergrupos (15 dias) demonstrou a diminuição entre as células inflamatórias: G1 e G3 ( $p<0.0001), G 1$ e G4 ( $p<0.0001)$ e G2 e G3 $(p<0.05)$.

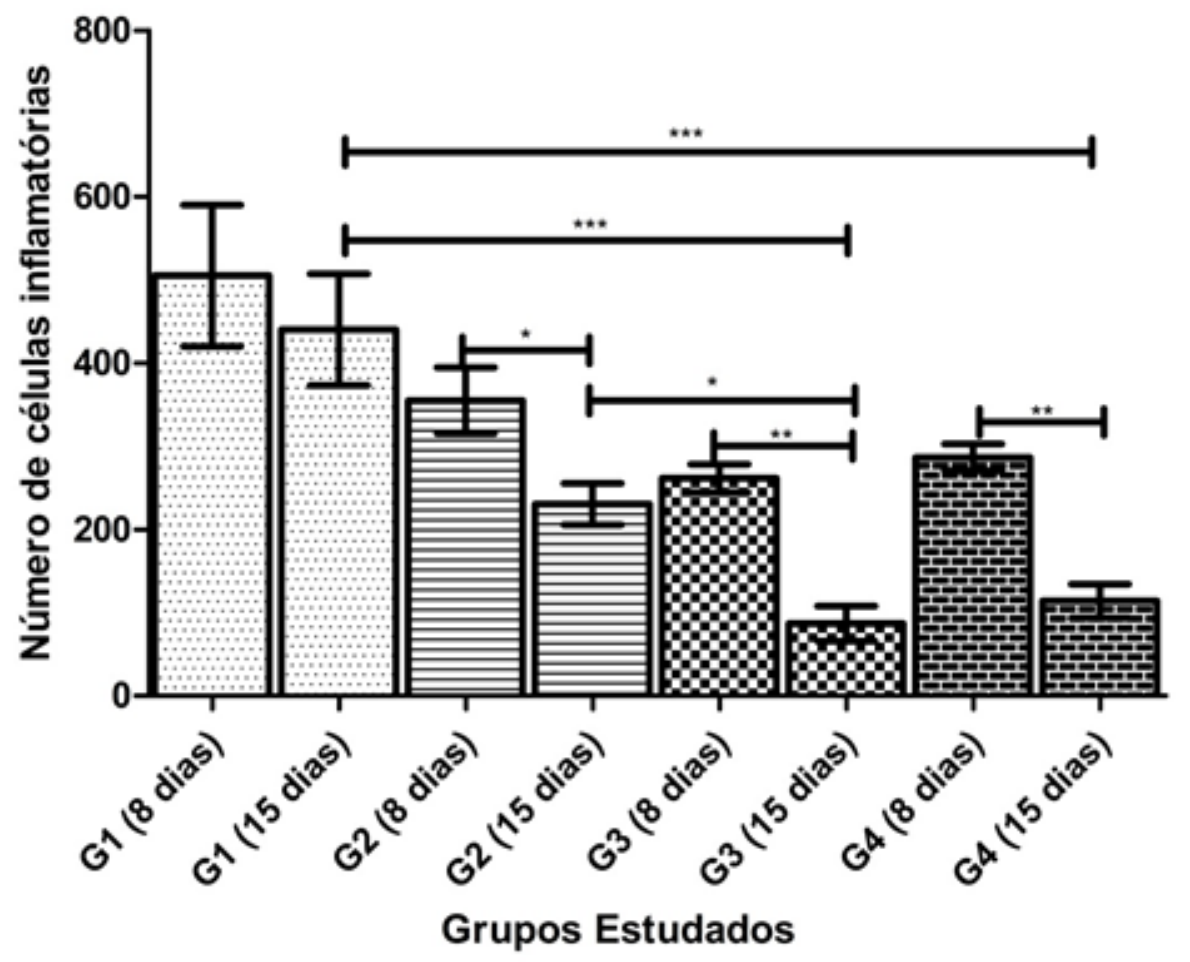

Figura 2. Resultados obtidos na análise intra e intergrupos acerca da contagem de células manuais dos grupos estudados. Legenda. G1 (Controle). G2 (Ultrassom pulsado (fonoforese) + gel das cascas do cajueiro (A. occidentale)). G3 (Gel das cascas do cajueiro (A. occidentale), uso tópico). G4 (Ultrassom pulsado). Fonte: Autores, 2019.

\section{DISCUSSÃO}

O presente estudo avaliou o efeito associado entre o gel à base das cascas do cajueiro (A. occidentale $L$.) e 0 ultrassom terapêutico sobre o processo de cicatrização de feridas cutâneas em camundongos. Foi comprovado com a realização desta pesquisa, que a UST, no modo pulsado, favoreceu resultados satisfatórios, em relação a diminuição no número de células inflamatórias. Oliveira et a. ${ }^{9}$ que enfatizaram que modalidade estudada quando comparada com 0 pulso contínuo, em fases iniciais de injúrias, apresenta resultados benéficos, uma vez que a irradiação contínua favorece o aquecimento local, fazendo com que haja a desorganização do material cicatricial, ocasionando efeitos deletérios na cicatrização.

Durante o processo de reparação tecidual, a síntese do colágeno é iniciada pelo incremento da ação dos 
fibroblastos, em que a deposição continuada do colágeno em áreas lesionadas contribui gradativamente para a estimulação do processo de cicatrização da ferida. Comparando-se o grupo controle com os tratados, tanto no $8^{\circ}$ dia quanto no $15^{\circ}$ dia pós-cirurgia, observou-se redução do número de células inflamatórias, o qual ficou mais evidente no $15^{\circ}$ dia nos grupos (G3 e G4), uma vez que após sete dias da lesão, o efeito de redução do processo inflamatório, associado ao estímulo celular (número de fibroblastos) e de síntese de colágeno acarretaram em um estímulo mais efetivo acerca do reparo tecidual em relação ao tratamento controle, já que os métodos empregados têm como finalidade acelerar o processo cicatricial, como demonstrado em diversos estudos $9,15,32$.

Yao et al. ${ }^{33}$ comprovaram em seu estudo que a UST favoreceu à redução das metaloproteinases de matriz de citocinas pró-inflamatórias, tais como: interleucinas (IL-6, IL-8, IL-1ß), fator de crescimento de transformação beta (TNF-a), fator estimulador de colônias de granulócitos e macrófagos (GM-CSF), fator de crescimento vascular derivado do endotélio (VEGF) e número de macrófagos, cujo quais possibilitaram a maior redução na porcentagem de redução de área da ferida.

A planta A. occidentale vem sendo utilizada em estudos científicos e demonstrados resultados terapêuticos satisfatórios, potencializando, em estudos preliminares, no processo de cicatrização ${ }^{34,37}$. Vários compostos existentes nesta planta têm demonstrado um potencial anti-inflamatório como, por exemplo, a presença de fenólicos (taninos e flavonoides $)^{38,39}$. Vasconcelos et al. ${ }^{23}$ enfatizaram que os flavonoides possuem capacidade de atuar sobre 0 sistema imunológico, tornando-se uma alternativa terapêutica promissora ao tratamento de processos inflamatórios, uma vez que o processo de cicatrização é um evento complexo envolvendo diversos mecanismos vasculares, celulares e bioquímicos, os quais são obviamente dependentes dos substratos nutricionais disponíveis, como por exemplo, a vitamina C que está diretamente relacionada à síntese de colágeno.

Os resultados encontrados neste estudo corroboram com Vasconcelos et al. ${ }^{23}$ Vanderlinde et al. ${ }^{35}$, MeloCavalcante et al..$^{36}$, os quais concluíram em suas pesquisas que o extrato a base da casca do cajueiro $A$. occidentale $L$, apresenta mediadores pró-inflamatórios, que favorece o restabelecimento tecidual (tecido de granulação e reepitelização), devido aos seus efeitos anti-inflamatórios, cicatrizante e antibacteriano, promovendo resultados positivos na cicatrização tecidual e, quando associado com recursos eletrofísicos, o UST, estimula a atividade fibrinolítica, acelerando a formação do tecido de granulação e a biossíntese de colágeno e elastina, obtendo um reparo mais efetivo ${ }^{9,15}$.

\section{CONSIDERAÇÕES FINAIS}

Conclui-se que o gel à base das cascas do cajueiro (A. occidentale L.) apresenta características farmacológicas importantes, as quais permitem classificar este recurso como um fitoterápico essencial para auxiliar no processo de 
reparação tecidual. É de suma importância ressaltar ainda que ambas as terapias (ultrassom pulsado associado ao gel à base das cascas do cajueiro, gel a base das cascas do cajueiro e ultrassom pulsado) na análise macroscópica, interferiram positivamente sobre a área de redução da ferida nas peles dos camundongos tratados, por ativação da síntese de colágeno, contribuindo para uma cicatrização mais efetiva, enquanto que a análise histológica demonstrou que os recursos (gel à base das cascas do cajueiro e ultrassom pulsado) apresentaram resultados expressivos quanto à modulação do processo inflamatório, sugerindo que os métodos foram mais efetivos sobre o reparo cicatricial.

\section{REFERÊNCIAS}

1. Korrapati PS, Karthikeyan K, Satish A, Krishnaswamy VR, Venugopal JR, Ramakrishna S. Recent advancements in nanotechnological strategies in selection, design and delivery of biomolecules for skin regeneration. Materials Science and Engineering: C. 2016; 67: 747-765.

2. Pessoa DR, Costa DR, Costa DR, Delpasso CA, Arisawa EÂLS. Efeitos da terapia laser de baixa intensidade em modelo experimental de tendinopatia em ratos: revisão de literatura. Revista Univap. 2017; 23(43): 102-112.

3. Nascimento LDES, Nascimento KFES, Pessoa DR, Nicolau RA. Effects of Therapy with Light Emitting Diode (LED) in the Calcaneal Tendon Lesions of Rats: A Literature Review. ScientificWorldJournal. 2019; 2019:6043019.

4. Wynn TA, Vannella KM. Macrophages in tissue repair, regeneration, and fibrosis. Immunity. 2016; 44(3): 450-462.

5. Vannella KM, Wynn TA. Mechanisms of organ injury and repair by macrophages. Annual review of physiology. 2017; 79: 593-617.

6. Montenegro EJN, Almeida BG, Alencar GG, Siqueira GR, Maia JN, Andrade Ma. Ação das ondas curtas pulsada na dor aguda induzida pela hipotermia: um estudo piloto. Revista brasileira de ciência e movimento. 2017;25(2):145-153.

7. Korelo RIG, Oliveira JJJD, Souza RSA, Hullek RDF, Fernandes LC. High frequency generator as treat- 
ment in scar pressure ulcers: pilot study. Fisioterapia em Movimento. 2013; 26(4), 715-724.

8. Korelo RIG, Valderramas S, Ternoski B, Medeiros DS, Andres LF, Adolph SMM. Aplicação da microcorrente como recurso para tratamento de úlceras venosas: um estudo piloto. Revista latino-americana de enfermagem. $2012 ; 20(4): 1-8$.

9. Oliveira PDD, Oliveira DA, Martinago CC, Frederico RCP, Soares CP, Oliveira RFD. Effect of low-intensity pulsed ultrasound therapy on a fibroblasts cell culture. Fisioterapia e Pesquisa. 2015; 22(2): 112-118.

10. Farcic TS, Baldan CS, Machado AFP, Caffaro LAM, Masson IFB Casarotto RA. Collagen Fibers in the Healing Process of Rat Achilles Tendon Rupture Using Different Times of Ultrasound Therapy. Advances In Wound Care. 2018; 7(4):114-120.

11. Tsai WC, Tang ST, Liang FC. Effect of therapeutic ultrasound on tendons. American Journal of Physical Medicine \& Rehabilitation. 2011; 90:1068-1073.

12. Ng GY. Comparing therapeutic ultrasound with microamperage stimulation therapy for improving strength of Achilles tendon repair. Connective Tissue Research. 2011; 52 (3):178-182.

13. Bertolini GRF, da Silva TS, Ciena AP, Artifon EL. Comparação do ultrassom pulsado e contínuo no reparo tendíneo de ratos. Fisioterapia \& Pesquisa. 2012; 19: 242-247.

14. Reuter P, Masomi J, Kuntze H, Fischer I, Helling K, Sommer C, Alessandri B, Heimann A, Gerriets T, Marx J, Kempski O, Nedelmann M. Low-frequency therapeutic ultrasound with varied duty cycle: effects on the ischemic brain and the inner ear. Ultrassound in Medicine Biology. 2010; 36(7): 1188-1195.

15. Draper DO. Facts and misfits in ultrasound therapy: steps to improve your treatment outcomes. European Journal of Physical and Rehabilitation Medicine 2014; 50:209-216.

16. Bruning MCR, Mosegui GBG, Vianna CMDM. The use of phytotherapy and medicinal plants in primary healthcare units in the cities of Cascavel and Foz do Iguaçu-Paraná: the viewpoint of health professionals. Ciencia \& 
saude coletiva. 2012; 17(10), 2675-2685.

17. Garcia LF, Lia RCC, Lopes RA, Oliveira DA, Souza FC, Santos HSL. Análise morfológica e morfométrica do tecido subcutâneo de ratos submetidos à ação de pasta de hidróxido de cálcio e óleo de Ricinus communis. Brazilian Dental Science. 2008; 11(3):47-54.

18. Martins MD, Marques MM, Bussadori SK, Mesquita-Ferrari RA, Pavesi VCS, Wadt NS, Fernandes KP. Citotoxicidade in vitro de extratos de arnica brasileira (Solidago microglossa) e arnica paulista (Porophyllum ruderale). ConScientiae Saúde. 2009; 8(1), 99-104.

19. Freitas, V. S., Rodrigues, R. A. F., \& Gaspi, F. O. G. (2014). Propriedades farmacológicas da Aloe vera (L.) Burm. f. Revista brasileira de plantas medicinais. 2014; 16(2), 299-307.

20. Sherif, N. H. (2014). Influence of Tropaeolum majus leaves extract in ameliorating damage induced by gamma radiation in rats. The Egyptian Journal of Hospital Medicine, 31(989), 1-8.

21. De Sousa JL, Costa PDL, Maia Filho ALM, Batista NJC, Uchoa VT, Dos Santos Alves W (2015). Análise da ação do gel da Bryophyllum calycinum Salisb (folha santa) associado ao ultrassom na cicatrização de feridas cutâneas. ConScientiae Saúde. 2015; 14(2): 213-221.

22. MARTINI, C. A. N., SCAPINI, J. G. S., COLLAÇO, L., MATSUBARA, A., \& VEIGA JÚNIOR, V. F. D. (2016). Comparative analysis of the effects of Copaifera multijuga oil-resin and nitrofurazona in the cutaneous wound healing process. Revista do Colégio Brasileiro de Cirurgiões. 2016; 43(6), 445-451.

23. Vasconcelos MS, Gomes-Rochette NF, de Oliveira ML, Nunes-Pinheiro DC, Tomé AR, Maia de Sousa FY, Pinheiro FG, Moura CF, Miranda MR, Mota EF, de Melo DF. Anti-inflammatory and wound healing potential of cashew apple juice (Anacardium occidentale L.) in mice. Experimental Biology And Medicine. 2015; 240(12): 1648-1655.

24. De Araujo S, Sousa IJO, Gonçalves RLG, De Sousa França AR, Dos Santos Negreiros P, Da Silva Brito AK, De Sousa Lima EB. Aplicações Farmacológicas e Tecnológicas da Goma do Cajueiro (Anacardium Occidentale L)um Produto Obtido da Flora Brasileira. Revista GEINTEC-Gestão, Inovação e Tecnologias. 2018; 8(1), 4292-4305. 
25. Barros SB, Leite CM, de Brito AC, Dos Santos Júnior JR, Zucolotto V, Eiras C. Multilayer Films Electrodes Consisted of Cashew Gum and Polyaniline Assembled by the Layer-by-Layer Technique: Electrochemical Characterization and Its Use for Dopamine Determination. International Journal Of Analytical Chemistry. 2012; 2012: 1-10.

26. Okpashi VE, Bayim BPR, Obi-Abang M. Comparative Effects of Some Medicinal Plants: Anacardium occidentale, Eucalyptus globulus, Psidium guajava,andXylopia aethiopicaExtracts in Alloxan-Induced Diabetic Male Wistar Albino Rats. Biochemistry Research International. 2014; 2014: 1-13.

27. Carvalho KC, Nicolau RA, Maia ALM, Barja PR, Sá HP, Santo LAE, Rocha GM. Estudo da resistência cicatricial cutânea de ratos tratados com fototerapia a laser. Conscientiae saúde. 2010; 9(2): 179-186.

28. Moura Júnior MJ, Maia Filho AL, Pessoa DR, Alves MD, Justino JS, Andrade MS, Rebêlo AM, de Lima CJ, Pinheiro AL, Silveira $L$ Jr. Assessing the biochemical changes of tendons of rats in an experimental model of tenotomy under therapeutic ultrasound and LEDs (625 and 945) by near-infrared Raman spectroscopy. Lasers in Medical Science. 2015; 30(6): 1729-1738.

29. Matos FJA. Introdução à fitoquímica experimental. 3 ed. Fortaleza: Editora UFC, 2009.

30. Leal SS, Uchôa VT, Figuerêdo-Silva J, Soares RB, Mota DM, Alencar RC, Maia Filho ALM, Sant'Ana AEG, Beltrame Junior M Eficácia da fonoforese com ximenia americana I. Na inflamação de tendão de ratos. Revista brasileira de medicina do esporte. 2016; 22(5): 355-360.

31. Martins NLP, Malafaia O, Ribas-Filho JM, Heibel M, Baldez RN, Vasconcelos PRL, Moreira Hamilton, Mazza M, Nassif PAN. Análise comparativa da cicatrização da pele com o uso intraperitoneal de extrato aquoso de Orbignya phalerata (babaçu). Estudo controlado em ratos. Acta Cirúrgica Brasileira. 2006; 21(3): 66-75.

32. Nogueira VC, Coelho NP, Barros TL, Silva SM, Martins M, Arisawa EA. Biomodulation effects of LED and therapeutic ultrasound combined with semipermeable dressing in the repair process of cutaneous lesions in rats. Acta Cirurgica Brasileira. 2014; 29(9): 588-595.

33. Yao M, Hasturk H, Kantarci A, Gu G, Garcia[Lavin S, Fabbi M, Driver VR. A pilot study evaluating non』 
contact lowlfrequency ultrasound and underlying molecular mechanism on diabetic foot ulcers. International wound jornal. 2014; 11(6): 586-593.

34. Melo-Cavalcante AAa, Picada JN, Rubensam G, Henriques JAP. Antimutagenic activity of cashew apple (Anacardium occidentale Sapindales, Anacardiaceae) fresh juice and processed juice (cajuína) against methyl methanesulfonate, 4-nitroquinoline N-oxide and benzo[a]pyrene. Genetics and Molecular Biology. 2008; 31(3): 759-766.

35. Vanderlinde FA, Landim HF, Costa EA, Galdino PM, Maciel MAM, Anjos GC, Malvar DC, Côrtes WS, Rocha FF. Evaluation of the antinociceptive and anti-inflammatory effects of the acetone extract from Anacardium occidentale L. Brazilian. Brazilian Journal of Pharmaceutical Sciences. 2009; 45: 437-42.

36. Melo-Cavalcante AA, Dantas SMM de M, Leite A de S, Matos LA, e Sousa JM, Picada JN, da Silva J.In vivo antigenotoxic and anticlastogenic effects of fresh and processed cashew (Anacardium occidentale) apple juices. Journal of Medicinal Food. 2011; 14: 792-98.

37. Gordon A, Friedrich M, da Matta VM, Herbster Moura CF, Marx F. Changes in phenolic composition, ascorbic acid and antioxidant capacity in cashew apple (Anacardium occidentale L.) during ripening. Fruits. 2012; 67: 267-76.

38. Serafini M, Peluso I, Raguzzini A. Flavonoids as anti-inflammatory agents. Proceedings of the Nutrition Society. 2010; 69: 273-8.

39. Veitch NC, Grayer RJ. Flavonoids and their glycosides, including anthocyanins. Natural Product Reports. 2011; 28: 1626-95. 